Supporting Information

Additive-Assisted Interfacial Engineering for Efficient Carbon-Based

Perovskite Solar Cells Incorporated Dopant-Free Polymeric Hole

\title{
Conductor PBDT(S)-T1
}

Bingbing Fan,,$^{\dagger,+}$ Tingting You, ${ }^{\dagger}$ Kexiang Wang, ${ }^{\dagger}$ Ran Yin,,$^{\dagger}$ Yukun Gao, ${ }^{\dagger}$ Lijun

Huo, ${ }^{*}+$ and Penggang Yin* ${ }^{\dagger}$

${ }^{\dagger}$ School of Chemistry, Beihang University, Beijing 100191, China.

'School of Physics, Beihang University, Beijing 100191, China.

*Corresponding author.

E-mail address: pgyin@buaa.edu.cn (P.Yin); huolijun@buaa.edu.cn(L.Huo). 


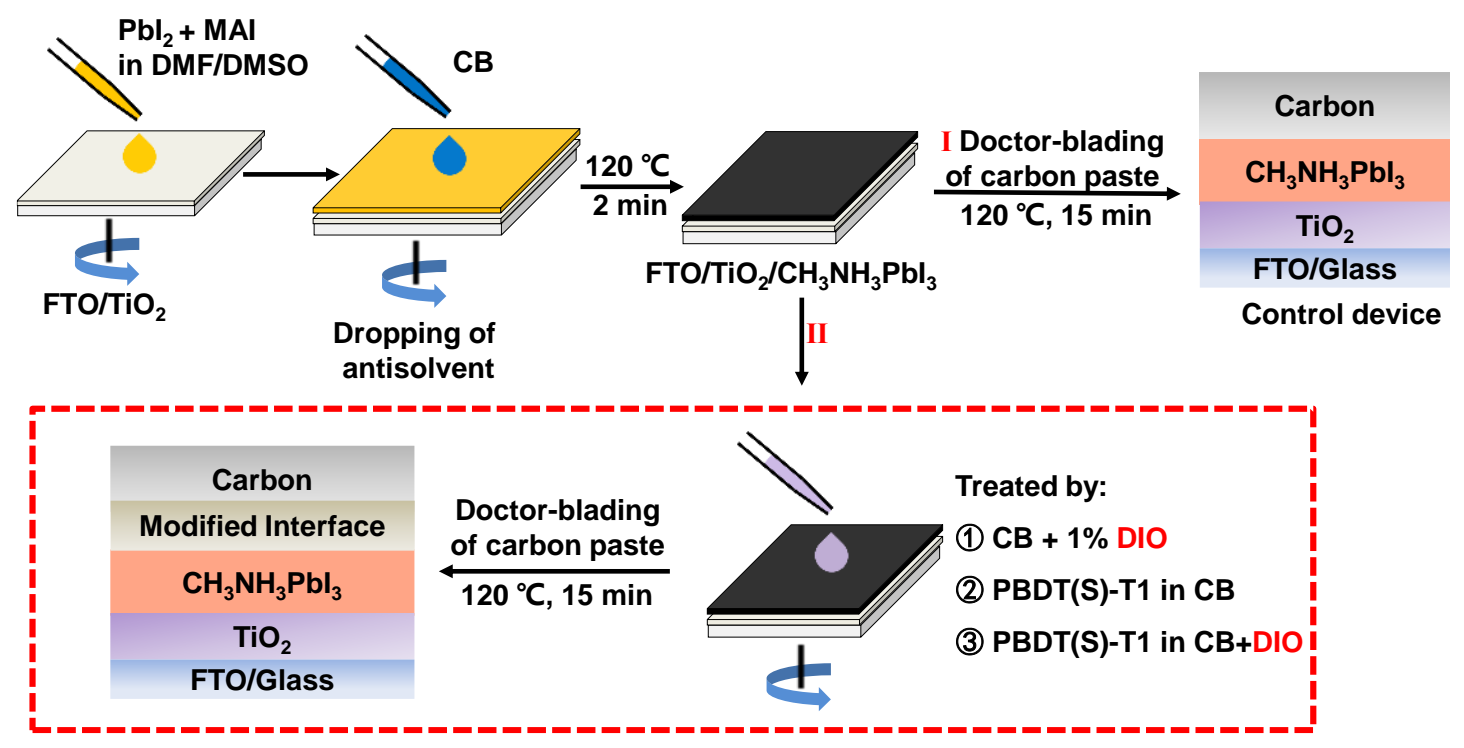

Schematic S1. The preparation procedure of PSCs investigated in this study. In procedure II, modified perovskite layers were annealed at $120{ }^{\circ} \mathrm{C}$ for $2 \mathrm{~min}$ before the doctor-blading of carbon electrode.

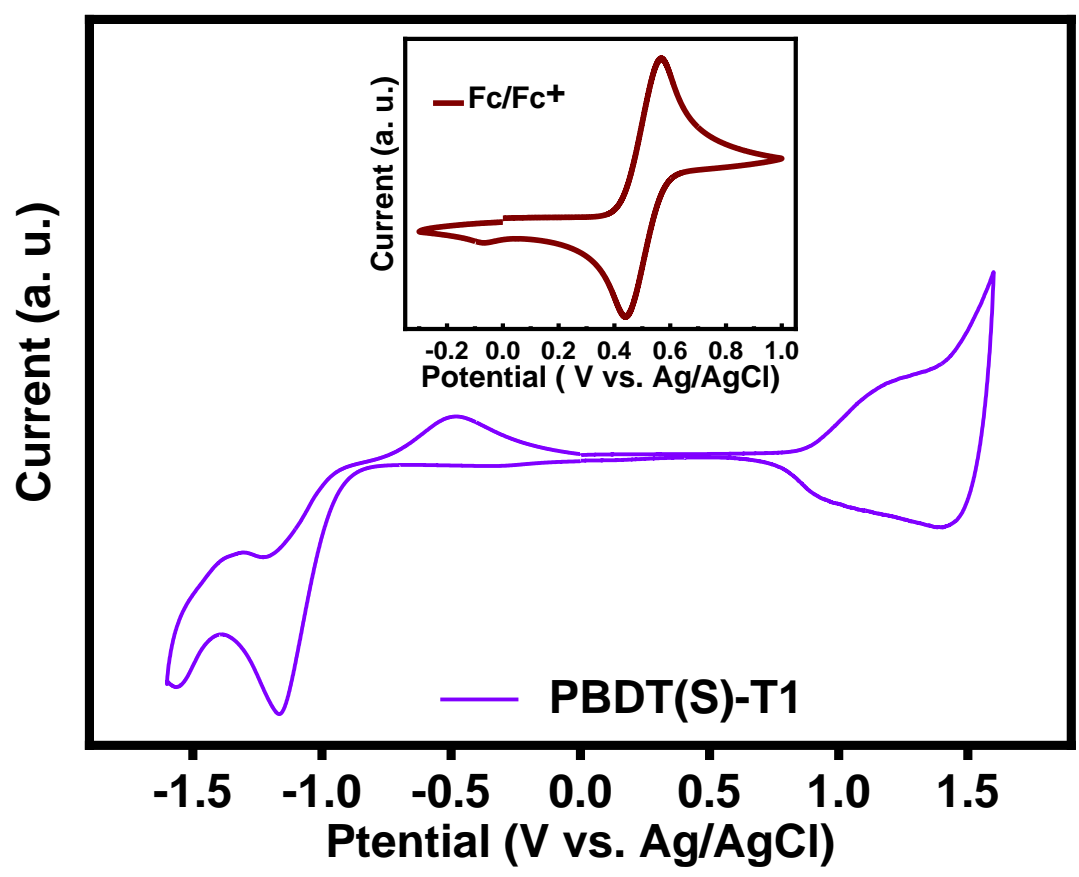

Figure S1. Electrochemical cyclic voltammetry curves of $\mathrm{Fc} / \mathrm{Fc}^{+}$and $\mathrm{PBDT}(\mathrm{S})-\mathrm{T} 1$. 


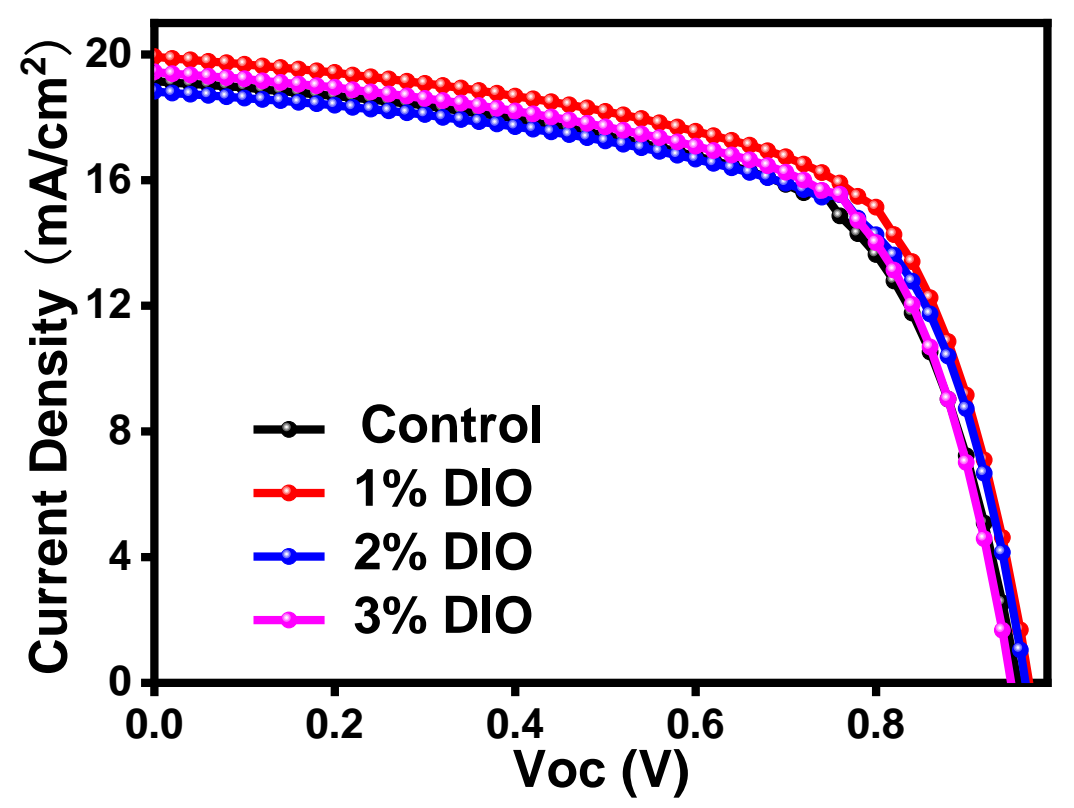

Figure S2. J-V characteristic curve of devices based on perovskite layer treated by different concentration of DIO.

Table S1. Photovoltaic performance parameters of control device devices based on perovskite layer treated by different concentration of DIO.

\begin{tabular}{ccccc}
\hline & $\begin{array}{c}J_{S C} \\
\left(\mathrm{~mA} / \mathrm{cm}^{2}\right)\end{array}$ & $\begin{array}{c}\text { Voc } \\
(\mathrm{V})\end{array}$ & $\begin{array}{c}\text { FF } \\
(\%)\end{array}$ & $\begin{array}{c}\text { PCE } \\
(\%)\end{array}$ \\
\hline- & 19.2 & 0.957 & 63.15 & 11.58 \\
$1 \%$ DIO & 19.9 & 0.970 & 62.66 & 12.11 \\
$2 \%$ DIO & 18.8 & 0.966 & 64.93 & 11.81 \\
$3 \%$ DIO & 19.4 & 0.950 & 63.99 & 11.82 \\
\hline
\end{tabular}



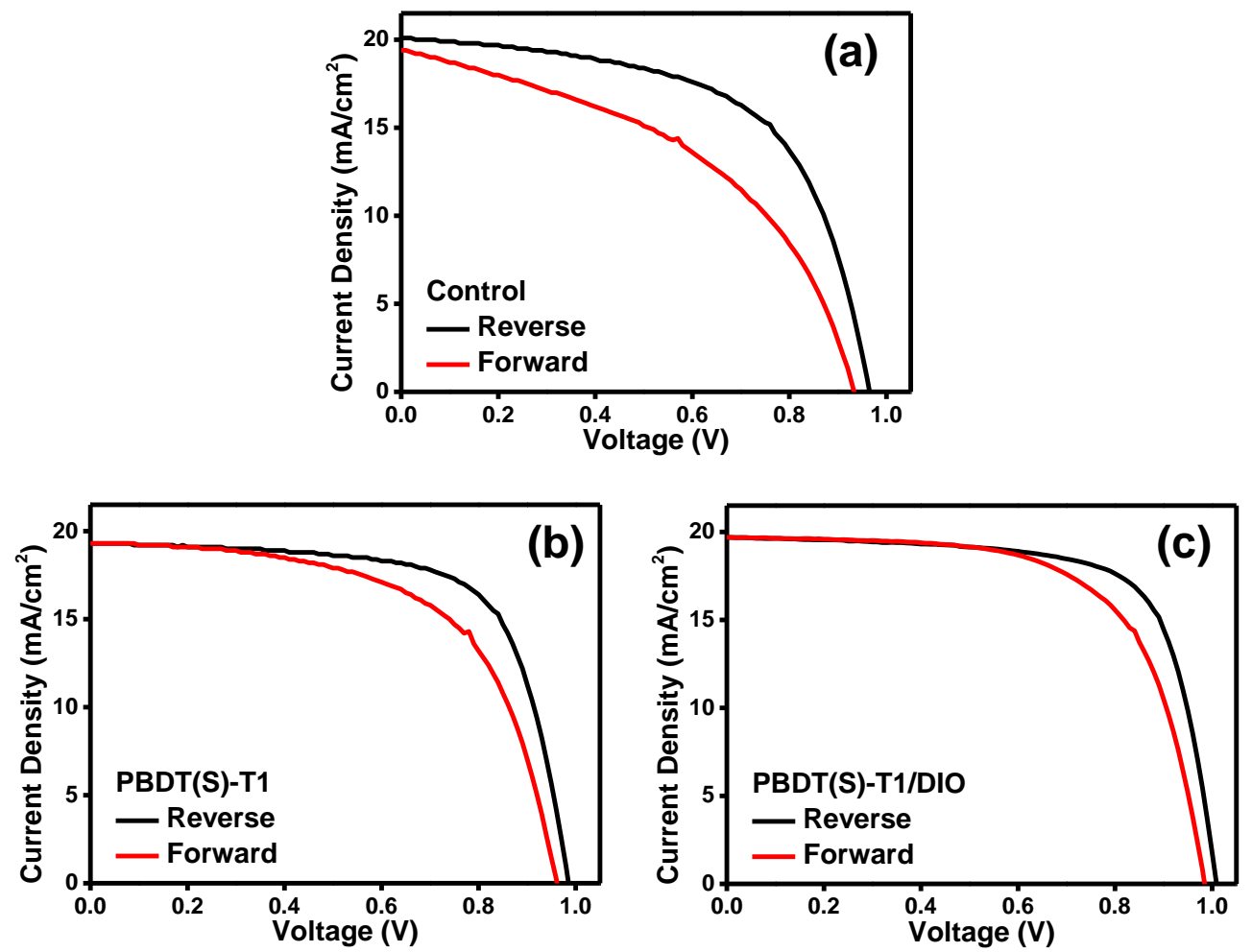

Figure S3. J-V curves obtained from different scanning direction for C-PSCs based on (a) pristine perovskite film, (b) PBDT(S)-T1 deposited and (c) PBDT(S)-T1/DIO deposited perovskite film, respectively.

Table S2. Photovoltaic performance parameters obtained from different scanning direction for C-PSCs based on pristine perovskite film, PBDT(S)-T1deposited and PBDT(S)-T1/DIO deposited perovskite film, respectively.

\begin{tabular}{ccccccc}
\hline \multirow{2}{*}{ Device } & $\begin{array}{c}\text { Scan } \\
\text { Direction }\end{array}$ & $\begin{array}{c}\text { Jsc } \\
\left(\mathrm{mA} / \mathrm{cm}^{2}\right)\end{array}$ & $\begin{array}{c}\text { Voc } \\
(\mathrm{V})\end{array}$ & $\begin{array}{c}\text { FF } \\
(\%)\end{array}$ & $\begin{array}{c}\text { PCE } \\
(\%)\end{array}$ & \multirow{2}{*}{ HI $^{\mathrm{a}}$} \\
\hline \multirow{2}{*}{ Control } & Reverse & 20.1 & 0.965 & 59.63 & 11.58 & \multirow{2}{*}{ ( } \\
& Forward & 19.4 & 0.933 & 45.34 & 8.22 & 0.29 \\
& Reverse & 19.3 & 0.985 & 68.92 & 13.12 & \multirow{2}{*}{ PBDT(S)-T1 } \\
& Forward & 19.3 & 0.961 & 59.90 & 11.12 & \\
PBDT(S)- & Reverse & 19.7 & 1.01 & 71.47 & 14.21 & \multirow{2}{*}{0.11} \\
T1/DIO & Forward & 19.7 & 0.984 & 64.80 & 12.57 & \\
\hline
\end{tabular}

${ }^{a}$ Hysteresis index $=\left(P C E_{\text {Reverse }}-P C E_{\text {Forward }}\right) / P C E_{\text {Reverse }}$ 
(a)

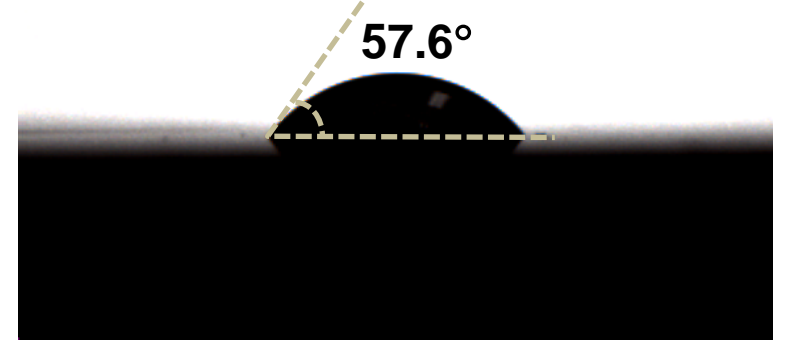

(b)

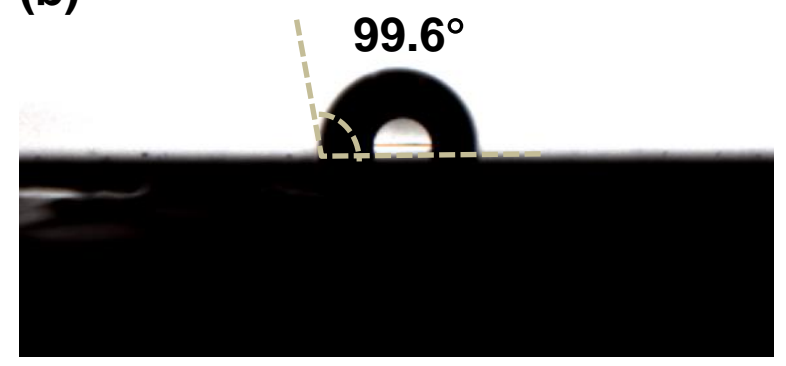

(c)

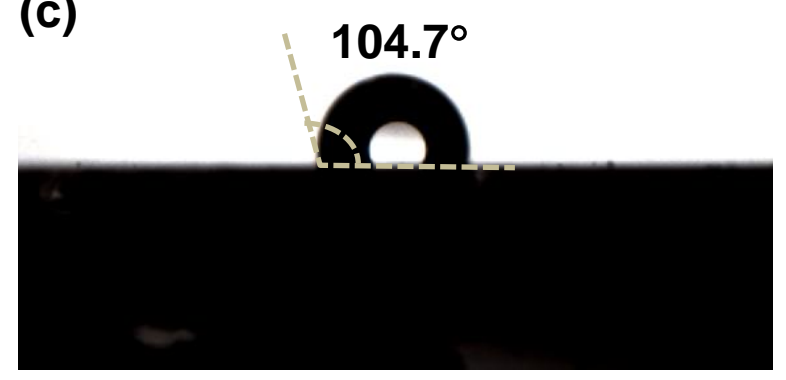

Figure S4. Contact angle images for droplets water on (a) perovskite film; (b) PBDT(S)-T1 deposited perovskite film; (c) PBDT(S)-T1/DIO deposited perovskite film.

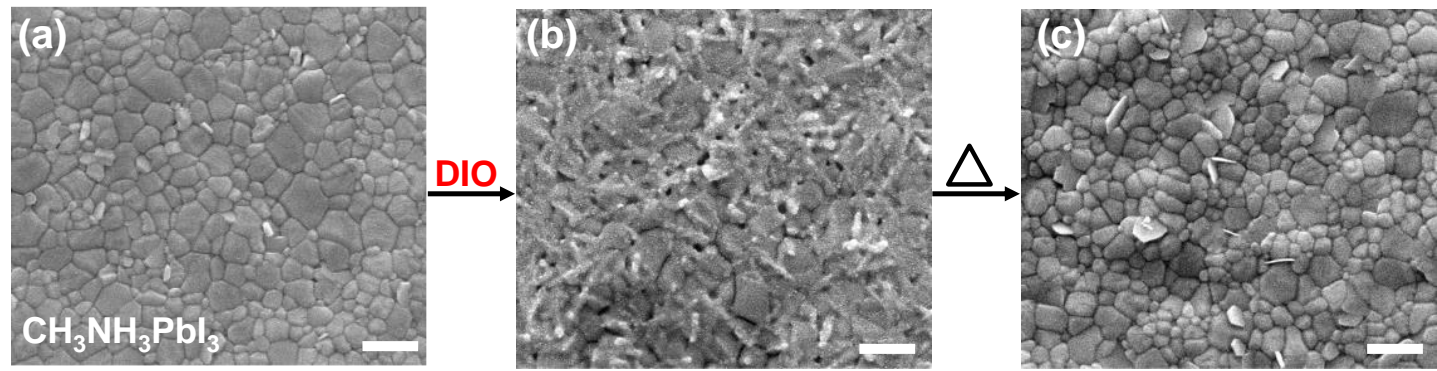

Figure S5. SEM images of (a) control perovskite film; DIO treated perovskite films (b) before and (c) after annealing. The sale bar is $1 \mu \mathrm{m}$. 


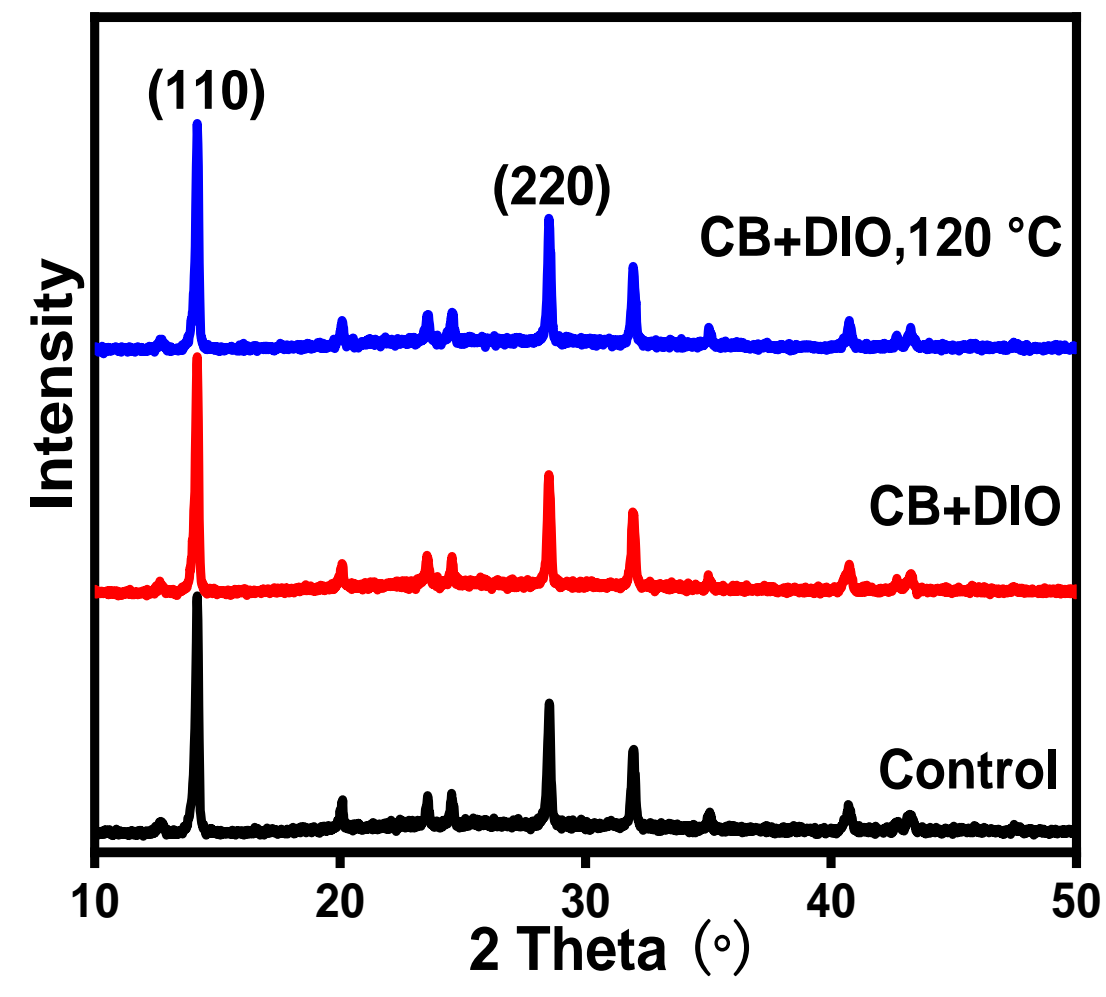

Figure S6. XRD pattern of control perovskite film (black line), DIO treated perovskite film before (red line) and after annealing (blue line).

Table S3. Structure and boiling point of CB and DIO.

\begin{tabular}{|c|c|c|}
\hline Liquid & Structure & Boiling point \\
\hline $\mathrm{CB}$ & \\
\hline DIO & & $132.2^{\circ} \mathrm{C}$ \\
\hline
\end{tabular}




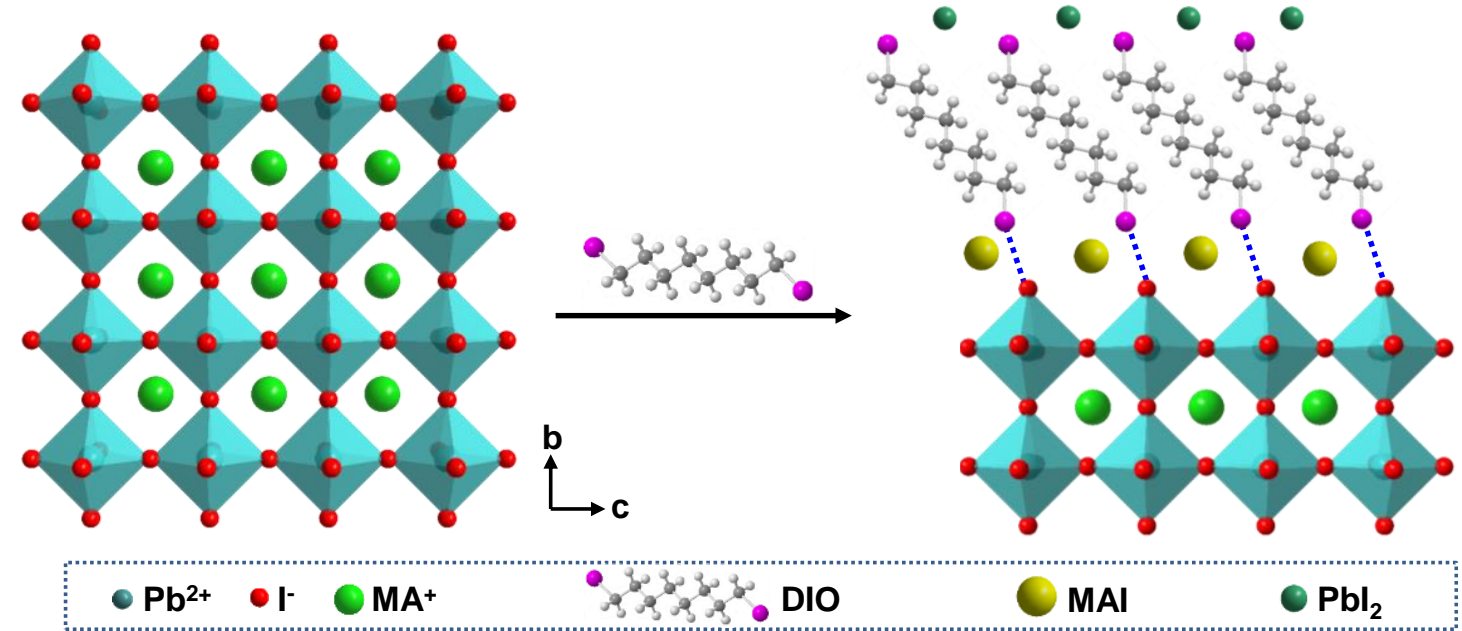

Figure S7. Schematic illustration of perovskite surface crystal evolution after the DIO treatment.
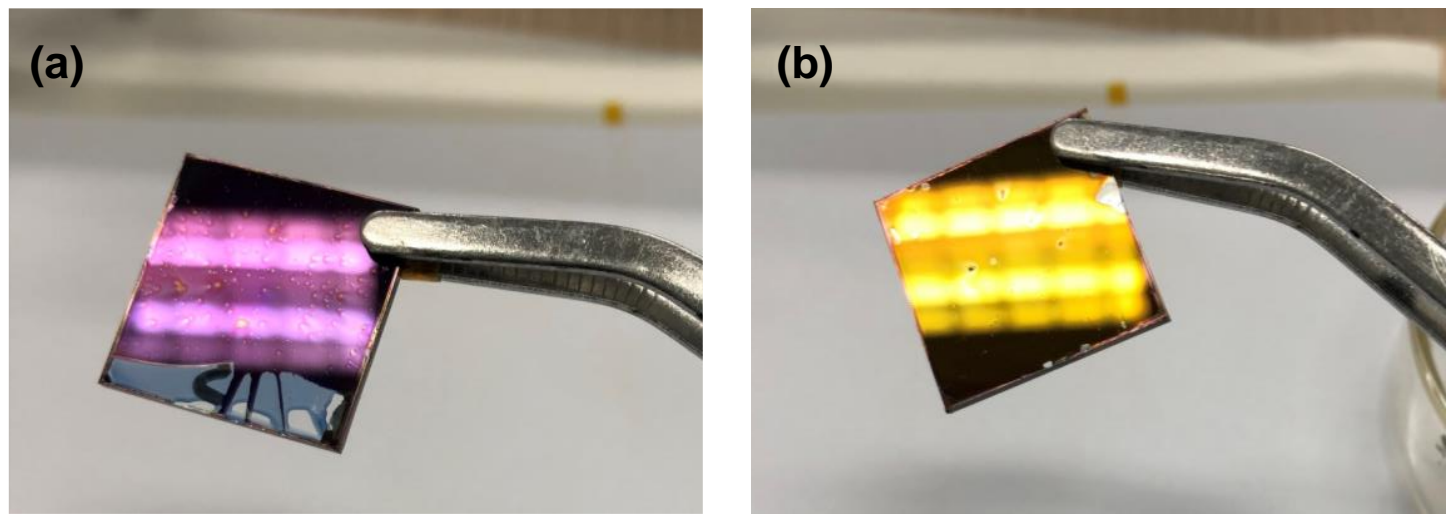

Figure S8. Photograph images of PBDT(S)-T1/DIO casted perovskite film (a) before and (b) after annealing.

Table S4. Fitted PL lifetimes $\left(\tau_{1}, \tau_{2}\right.$ and $\left.\tau\right)$ of films on $\mathrm{FTO} / \mathrm{TiO}_{2}$ substrates. The PL lifetimes $\left(\tau_{1}, \tau_{2}\right.$ and $\left.\tau\right)$ are fitted by the biexponential model: $\mathrm{y}=\mathrm{A}_{1} * \exp \left(\mathrm{x} / \tau_{1}\right)$ $+\mathrm{A}_{2}{ }^{*} \exp \left(\mathrm{x} / \tau_{2}\right), \mathrm{A}_{1}$ and $\mathrm{A}_{2}$ are refactor, $\tau_{1}$ and $\tau_{2}$ are the lifetimes of the fast and slow decay component, respectively. And: Fraction $1=A_{1} /\left(A_{1}+A_{2}\right) ;$ Fraction $2=A_{2} /\left(A_{1}+A_{2}\right)$ and $\tau=$ Fraction $1 * \tau_{1}+$ Fraction $2 * \tau_{2}$.

\begin{tabular}{cccccc}
\hline & $\tau_{1}(\mathrm{~ns})$ & Fraction 1 & $\tau_{2}(\mathrm{~ns})$ & Fraction 2 & $\tau(\mathrm{ns})$ \\
\hline Control & 26.07 & 0.50 & 26.07 & 0.50 & 26.07 \\
PBDT(S)-T1 & 0.52 & 0.96 & 11.91 & 0.04 & 0.92 \\
PBDT(S)-T1/DIO & 0.37 & 0.93 & 4.73 & 0.07 & 0.68 \\
\hline
\end{tabular}




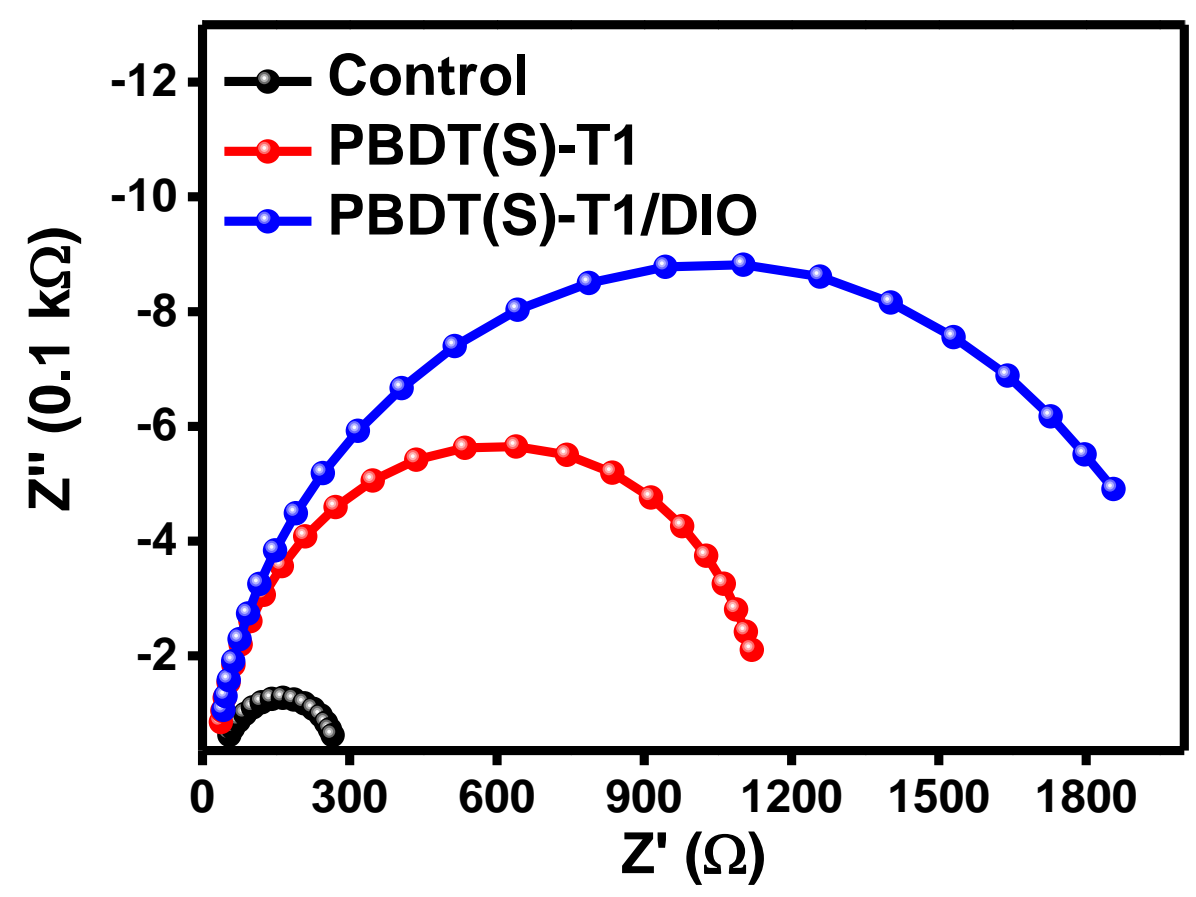

Figure S9. Nyquist plots of the perovskite solar cells measured in the dark. 